\title{
Is this ST-elevation because of myocardial ischaemia or a Brugada pattern? An interesting case review
}

\author{
Pankaj Garg • Thomas Nelson · Jonathan Sahu • \\ Paul Sheridan
}

Received: 19 August 2014/ Accepted: 27 August 2014/Published online: 5 September 2014

(C) SIMI 2014

Brugada syndrome, the combination of right bundle branch block, and right precordial ST-elevation complicated by sudden cardiac death, was first reported in 1992 [1]. Since then, multiple mutations in SCN5a, a gene encoding a subunit of the cardiac voltage-gated sodium channel $\mathrm{Na}_{\mathrm{v}} 1.5$, have been identified in patients with the characteristic electrocardiographical (ECG) changes [2].

The Brugada syndrome (BrS) was initially described in structurally normal hearts, and was considered a primary electrical condition, with structural abnormalities considered exclusion criteria for the diagnosis of BrS. With improved imaging modalities and greater awareness of the condition, a number of minor structural abnormalities of the right ventricle $(\mathrm{RV})$ have been described, in particular RV dilatation and RV wall-motion abnormalities [3].

The classical ECG changes may be dynamic, and are often only unmasked during inter-current febrile illness, ingestion of certain medications, electrolyte abnormalities and alcohol or cocaine toxicity [4]. However, ECG changes similar to those seen in BrS have been described in atypical right bundle branch block, hyperkalaemia, hypercalcaemia, acute myopericarditis, hypothermia and acute myocardial infarction [5]. In patients with acute myocardial infarction, these 'pseudo-Brugada' ECG changes would be expected to resolve after coronary re-vascularization.

P. Garg $(\bowtie) \cdot$ T. Nelson · J. Sahu · P. Sheridan

Department of Cardiology, Northern General Hospital, Sheffield

Teaching Hospitals NHS Foundation Trust, Herries Road,

Sheffield S5 7AU, UK

e-mail: pankajvic@gmail.com

J. Sahu $\cdot$ P. Sheridan

Department of Cardiovascular Science, University of Sheffield, Medical School, Beech Hill Road, Sheffield S10 2RX, UK
We present the case of a 44-year-old man with no past cardiac medical history or risk factors for coronary artery disease. He presented with a 2-month history of intermittent atypical chest pain and palpitations. There was no family history of significant ischaemic heart disease, dysrhythmia, structural heart disease, or sudden cardiac death. A resting 12-lead ECG revealed sinus rhythm with a 'type3' Brugada pattern with less than $1 \mathrm{~mm}$ ST-elevation in leads V1-V2 not meeting the required voltage criteria for the classical type-1 ECG pattern.

In view of the history of chest pain (a feature not normally associated with $\mathrm{BrS}$ ), the clinical team felt that the ECG abnormalities might represent 'pseudo'-Brugada changes secondary to acute ischaemia. Accordingly, a full cardiovascular workup including cardiac magnetic resonance imaging (CMR) and coronary angiography were performed. This revealed significant stenosis of the proximal right coronary artery (RCA). This was stented, and good flow results were obtained. Post-revascularization ECGs demonstrated persistent ST changes (Fig. 1a) that did not resolve after the coronary intervention. Subsequent provocation testing with Ajmaline (Class IC anti-dysrhythmic sodium channel blocking agent,) unmasked the classical type-1 Brugada pattern (Fig. 1b). The Cardiac Magnetic Resonance Imaging (CMR) revealed a mildly dilated $\mathrm{RV}$ with preserved systolic function.

This is an unusual case as it highlights the co-existence of the Brugada channelopathy with ischaemic heart disease in a young patient with low risk for coronary artery disease. Additionally, there was evidence of macroscopic structural disease seen on CMR, adding weight to the recent body of evidence suggesting that $\mathrm{BrS}$ is often associated with subtle structural abnormalities. Hence, in patients presenting to emergency department with ST-elevation without chest 
(a)
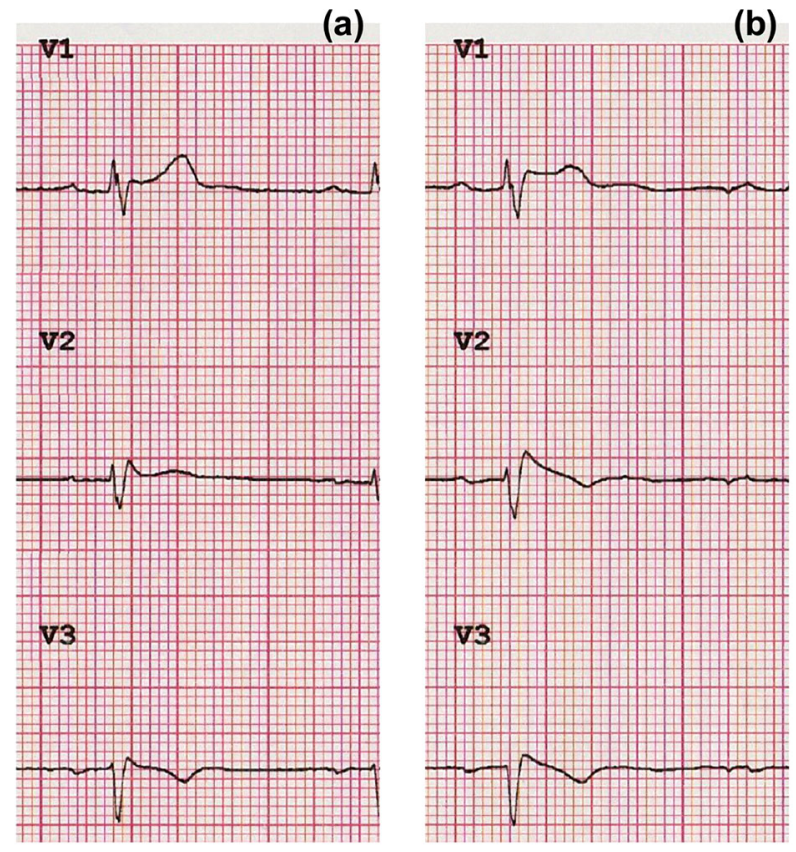

(b)

Fig. 1 Precordial ECG tracings pre (a) and post (b) Ajmaline provocation test

pain history, other differential diagnoses than ischaemia should be considered, and appropriate history and investigative strategy should be deployed.

\section{References}

1. Brugada P, Brugada J (1992) Right bundle branch block, persistent ST segment elevation and sudden cardiac death: a distinct clinical and electrocardiographic syndrome: a multicenter report. J Am Coll Cardiol 20(6):1391-1396

2. Kapplinger JD, Tester DJ, Alders M, Benito B, Berthet M, Brugada $J$ et al (2010) An international compendium of mutations in the SCN5A-encoded cardiac sodium channel in patients referred for Brugada syndrome genetic testing. Heart Rhythm 7(1):33-46

3. Papavassiliu T, Wolpert C, Fluchter S, Schimpf R, Neff W, Haase KK et al (2004) Magnetic resonance imaging findings in patients with Brugada syndrome. J Cardiovasc Electrophysiol 15(10): $1133-1138$

4. Antzelevitch C, Brugada P, Borggrefe M et al (2005) Brugada syndrome: report of the second consensus conference: endorsed by the Heart Rhythm Society and the European Heart Rhythm Association. Circulation 111(5):659-670

5. Brugada P, Benito B, Brugada R et al (2009) Brugada syndrome: update 2009. Hellenic J Cardiol 50(5):352-372 